\title{
PTSD and Physical Comorbidity Among Women Receiving Medicaid: Results From Service-Use Data*
}

\author{
Julia S. Seng \\ University of Michigan Institute for Research on Women and Gender, School of Nursing, \\ and Department of Obstetrics and Gynecology \\ M. Kathleen Clark and Ann Marie McCarthy \\ University of Iowa College of Nursing \\ David L. Ronis \\ Ann Arbor VA Medical Center and University of Michigan School of Nursing
}

\begin{abstract}
Patterns of physical comorbidity among women with posttraumatic stress disorder (PTSD) were explored using Michigan Medicaid claims data. PTSD-diagnosed women $(n=2,133)$ were compared with 14,948 randomly selected women in three health outcome areas: ICD-9 categories of disease, chronic conditions associated with sexual assault history in previous research, and reproductive health conditions. $P T S D$ was associated with increased risk of all categories of diseases (OR range $=1.3-4.8$ ), endometriosis $(O R=2.7)$, and dyspareunia $(O R=3.4)$. When PTSD was not complicated by other mental health conditions, odds ratios for chronic conditions ranged from 1.9 for fibromyalgia to 4.3 for irritable bowel. Comorbidity with depression or a dissociative or borderline personality disorder raised risk in a dose-response pattern.
\end{abstract}

The prevalence of posttraumatic stress disorder (PTSD) is estimated to be $4.6 \%$ among U.S. women, with a $12.3 \%$ lifetime rate (Resnick, Kilpatrick, Dansky, Saunders, \& Best, 1993). This disorder affects women at twice the rate of similarly traumatic-event-exposed men and becomes chronic in more than half of affected women (Breslau, Davis, Peterson, \& Schultz, 1997). Although women with PTSD have been less studied than men, they have reported worse health perceptions, more functional disability, and more physical symptoms (Andreski, Chilcoat, \& Breslau, 1998; Kimerling, Clum, \& Wolfe, 2000). Physical comorbidity with PTSD is believed to occur via psychological, biological, behavioral, and attentional mechanisms (Schnurr $\&$ Green, 2004). Biological sex, gender socialization, and menstrual cycle status may be factors in PTSD risk, morbidity, and treatment response as well as patterns of psychiatric and physical comorbidity (Rasmusson \& Friedman, 2002, Rasmusson et al., 2001). Current knowledge of

\footnotetext{
*This article was edited by the journal's previous editor, Dean G. Kilpatrick.

The authors acknowledge Sara Nichols, Ph.D., for her work on the early analytic decisions in this project and Rafatjan Abdul-Rasoul, M.S., for her assistance creating analytic files from the raw data. The Michigan Medicaid data were made available by Health Management Associates, Inc., Lansing, MI, where we acknowledge the expert support of Dennis Roberts, Ph.D. This project was completed while Dr. Julia S. Seng was a Pfizer Postdoctoral Fellow.

Correspondence concerning this article should be addressed to: Julia S. Seng, University of Michigan Institute for Research on Women and Gender, Lane Hall, Ann Arbor, MI 48109-1290. E-mail: jseng@umich.edu.

(C) 2006 International Society for Traumatic Stress Studies. Published online in Wiley InterScience (www.interscience.wiley.com) DOI: 10.1002/jts.20097
} 
physical comorbidity with PTSD among women is based on survey studies of health perceptions (e.g., Kimerling et al., 2000) and case-control studies of patients of both genders with specific physical conditions such as irritable bowel syndrome (e.g., Irwin et al., 1996), or small samples of medical patients who often are veterans (e.g., Ouimette et al., 2004).

The goal of this analysis was to explore patterns of physical comorbidity with PTSD among a population of women via descriptive epidemiological analysis of Medicaid service-use data. The analysis focused on three sets of health outcomes: categories of disease, five chronic conditions (i.e., chronic fatigue, fibromyalgia, irritable bowel, chronic pelvic pain, and menstrual pain) associated in numerous previous studies with a history of sexual abuse or assault, and reproductive problems. This analysis considered some of the additional elements contributing to the complexity of this phenomenon, to the extent feasible with service-use data. We controlled for the effects of sex and gender (by limiting the study to women), poverty and access to health care (by studying Medicaid recipients and controlling for extent of coverage), age and reproductive stage of life (by stratifying into reproductive-age and postmenopausal-age groups), psychiatric comorbidity (by controlling for depression and other psychiatric diagnoses and differentiating more severe cases of PTSD, those that have depressive comorbidity, and those that likely have complex PTSD), and recent victimization (discerned via coding for victim services).

\section{METHOD}

\section{Design and Data Source}

This is a case-control analysis of an existing public health insurance health-service-use database comparing every adult female recipient with the PTSD diagnostic code ( $n=2,133$ cases) and 14,948 randomly selected comparison women recipients without a PTSD diagnosis. The data are from the state of Michigan's Medicaid program. This database is in the public domain and was made available for research as a delimited dataset, under a data-use agree- ment, consistent with Health Insurance Portability and Accountability Act regulations (HIPAA, 1996) and with Institutional Review Board approval, by Health Management Associates of Lansing Michigan.

Medicaid is the public program providing health insurance coverage for people who live in poverty and do not have private insurance. Eligibility is based on demonstrated need and must be periodically renewed. The Medicaid eligibility data contain demographic information limited to date of birth, gender, self-reported race, county of residence, and postal code area. They also indicate dates of coverage, type of program (fee-for-service or capitated), and general reason for public insurance eligibility, including disability, but with no information about the type or cause of disability. The claims databases include numerical coding for diagnoses [International Classification of Diseases, 9th ed. (ICD-9); Practice Management Information Corporation, 1998], procedures (Current Procedural Terminology, CPT), and drugs (National Drug Classification) for purposes of processing payment to institutions and practitioners for inpatient, ambulatory, and pharmacy services. They include mental health services delivered by providers eligible by state law to provide services including primary care physicians and advance practice nurses, social workers, psychologists, and psychiatrists. Norms dictate that the diagnoses recorded be a focus of the hospitalization or encounter, whether tentative or confirmed.

The data in this analysis are for 1994-1997 Medicaidcovered services for recipients who had coverage exclusively from a fee-for-service program. Capitated (monthly flatfee program) records were eliminated because Michigan's capitated Medicaid programs have "mental health carve outs," whereby physical health services are capitated (i.e., not coded in detail), but mental health services are charged in the traditional "fee-for-service" manner. This results in cases where mental health diagnoses exist in the record, but no medical diagnoses appear, requiring exclusion of these cases from this analysis. The first year when the cleaned tables are available for the full population of recipients is 1994. After 1997, the proportion of capitated cases became high enough to introduce a sampling bias since healthier people are more likely to change coverage and move to 
HMO-style programs (D. Roberts, Health Management Associates, personal communication, September 6, 2001). There is no way to know what, if any, services recipients used beyond the services covered by Medicaid, but among poor people, the proportion of health care services paid for out-of-pocket is likely to be small.

\section{Sample and Power Analusis}

Claims records for every female recipient with the ICD-9 diagnostic code for PTSD $(n=4,894)$ were extracted out of the entire female Michigan Medicaid population (approximately 900,000 enrolled per year) across the 1994 through 1997 time period to determine the prevalence of the PTSD diagnosis for females of all ages ( $0-94$ years old). To achieve adequate power for low-prevalence conditions, we wanted at least a 3:1 ratio of comparison to case records (Hulley et al., 2001) across all age groups, including the late adolescent peak for PTSD (Breslau et al., 1997). Thus, we extracted comparison records via random number assignment from all remaining females in a 10:1 ratio $(n=48,940)$. After excluding records with any months in capitated programs, 3,816 PTSD case records and 27,366 comparison records remained, and the desired 3:1 ratio was achieved in all age strata. This report focuses on the analysis for adults in two age strata: reproductiveage (18-54 years) and postmenopausal-age (>54 years) women with results for children and adolescents (Seng, Graham-Bermann, Clark, McCarthy, \& Ronis, 2005) and a health disparities analysis (Seng, Kohn-Wood, \& Odera, 2005) reported elsewhere.

Power analysis was done with the PASS 2000 software program (Cohen, 1988; Hintze, 2000). To determine sample size required, we assumed at least $10 \%$ in the PTSD case group, a two-tailed alpha of .05, and power of $80 \%$. With a conservative odds ratio (2.4) and $25 \%$ of variance explained by other predictors, the sample size needed to test the lowest prevalence disorder $(2 \%)$ is 3,600. Within the postmenopausal-age group ( $>55)$, the sample size was 3,325, but this includes only 44 women with the PTSD diagnosis, so descriptive and bivariate analyses only were conducted for that group.

\section{Data Analusis}

Demographic variables available included gender, age, ethnicity, and program and coverage information from the eligibility table. Most other variables were created out of inpatient and practitioner claims tables from individual codes (e.g., PTSD Code 309.81) or ranges of codes (e.g., infectious disease Codes 001-139) in the ICD-9 taxonomy (Practice Management Information Corporation, 1998). The victimization and mental health treatment variables were created from visit codes (e.g., for counseling with a victim of abuse), explanation codes (e.g., explaining the means of an injury to be battering), procedure codes (e.g., psychotherapy), and pharmacy codes (e.g., drug class).

Bivariate case-control analyses of prevalence rates and odds ratios were done using $t$ test or chi-square. These comparisons were done by age strata with range of ages determined based on likely reproductive status because several of the conditions of interest occur primarily prior to menopause (e.g., menstrual pain, endometriosis) or after menopause (e.g., cardiac disease, osteoporosis).

A series of multivariate logistic regression models were specified to determine the effect of PTSD exposure on the health outcomes using a limited number of covariates. Choice of covariates was made using a change-in-estimate approach (Greenland, 1989). The effect of age and reproductive status is addressed by stratifying, limiting multivariate analysis to reproductive-age women only. Two other covariates, race and exposure to insurance, were included (exposure to insurance) or excluded (race/ethnicity) based on the Mantel-Haenszel test of conditional independence and the Breslow-Day test of homogeneity of the odds ratios to assess confounding and effect modification, respectively (Agresti, 1990; Breslow \& Day, 1993). The hospital victim-service code was selected as a covariate a priori because it is an important potential alternative explanation for the outcomes. The primary predictor in the multivariate analysis was a six-category mental health variable that divides the comparison and case groups into three categories each. Comparison women are designated as having 
(a) no mental health diagnosis, (b) a mental health diagnosis that is neither PTSD nor depression, or (c) depression without PTSD. The case group is designated as having (d) PTSD without depression, (e) PTSD with depression, or (f) PTSD (with or without depression) in conjunction with either a dissociative or a borderline personality disorder diagnosis. This predictor controls for psychiatric comorbidity and addresses the alternative hypotheses that it is not PTSD, perse, but rather having depression, having a mental disorder in general, or having only severe PTSD that is associated with these adverse health outcomes. These models were estimated in a hierarchical manner, with the coverage and victimization variables entered first and then the PTSD categorical variable, to compare the effects of victimization alone and then victimization and PTSD together. The adjusted odds ratios were calculated for all three sets of health outcomes.

\section{RESULTS}

\section{Demographic and Psychiarric Characteristics}

The annual prevalence of PTSD diagnosis among all female (child and adult) Michigan Medicaid recipients was $0.45 \%$ in 1994 and rose to $0.50 \%$ in 1997 . Table 1 describes the demographic, health services, and psychiatric characteristics of the PTSD and comparison groups by age strata. Among postmenopausal recipients, the mean age of the PTSD-diagnosed group was 10 years younger than the comparison group (62 vs. 72$), F(1,3323)=$ $42.7, p<.001$. While statistically significant, the mean age of PTSD-diagnosed recipients in the reproductive-age stratum was only slightly older (1 year) than recipients in the comparison group. Among reproductive-age women, the PTSD-diagnosed group had more months of coverage. PTSD-diagnosed women in the postmenopausal group did not differ with regard to coverage. PTSD-diagnosed women in both age strata were twice as likely to be eligible for Medicaid due to disability. African American women were only $11.9 \%$ of the reproductive-age PTSD-diagnosed recipients while they represent $28.2 \%$ of that comparison group, $\chi^{2}(1, N=13,756)=248.1, p<.001$, a differ- ence which has been studied in a separate analysis (Seng, Kohn-Wood, \& Odera, 2005).

Among the 13,756 recipients in the reproductive-age group, coding for hospital encounters related to victimization occurred 320 times, with $9.1 \%$ of the PTSD group and $1.1 \%$ of comparison recipients having hospital episodes due to inflicted injury, or rape, $\chi^{2}(1, N=13,756)=$ 496.7, $p<.001$. Rates of victimization coding ranged from $0.6 \%$ among those with no mental health diagnosis to $4.4 \%$ with depression but not PTSD, to $5.3 \%$ with PTSD but not depression, and to $19.5 \%$ among those with complex PTSD. One third of the codes for rape or inflicted injury occurred after the first date of the PTSD diagnosis code, indicating a potential revictimization.

In the randomly selected comparison group, the prevalence of any and all non-PTSD psychiatric codes in the ICD-9 taxonomy range 290 to 319 (including, thus, those with organic conditions such as dementia and mental retardation) was $27 \%$ in the reproductive-age stratum and $40.3 \%$ among the postmenopausal women. Prevalence of mental health disorders considered to be comorbid with PTSD or its associated features were higher in the PTSD case group, as were two severe mental illnesses (i.e., bipolar disorder and schizophrenia, see Table 1). Odds of suicide, attempted suicide, and self-inflicted injury were 18.1 times as great in the PTSD-diagnosed group (0.2 vs. $3.7 \%$ prevalence, $95 \% \mathrm{CI}=11.5,28.4$ or 11.5-28.4).

To consider PTSD chronicity, we used the three mental health diagnosis subgroups within the PTSD case group: (a) those who had a PTSD diagnosis but no depression, dissociation, or borderline personality comorbidity, or "simple PTSD;" (b) those who had codes for both PTSD and depression; and (c) those who had PTSD and also either a dissociative disorder or a borderline personality disorder diagnosis, or "complex PTSD." This subgroup categorizing of PTSD-diagnosed women showed a dose-response pattern for length of duration of the PTSD code appearing in the data (see Table 1). Duration of PTSD coding likely reflects duration of PTSD treatment, which can be affected by resolution of the condition, treatment dropout, loss of coverage, or shift of focus of treatment away from PTSD. 
Table 1. Demographic and Psychiatric Characteristics of the Two Age Strata by PTSD Case or Comparison Group Status

\begin{tabular}{|c|c|c|c|c|}
\hline & \multicolumn{2}{|c|}{ Reproductive Age: $18-54$} & \multicolumn{2}{|c|}{ Postmenopause: $55+$} \\
\hline & Comparison & PTSD & Comparison & PTSD \\
\hline \multicolumn{5}{|l|}{ Age } \\
\hline Mean & 30 & $31^{* * *}$ & 72 & $62^{* * *}$ \\
\hline Median & 28 & 30 & 71 & 60 \\
\hline Mode & 20 & 30 & 70 & 55 \\
\hline \multicolumn{5}{|l|}{ Medicaid coverage } \\
\hline Mean number of months & 22 & $34^{* * *}$ & 32 & 36 \\
\hline Percent with continuous coverage & $9.8 \%$ & $24.9 \% * * *$ & $31 \%$ & $45.5 \% *$ \\
\hline \multicolumn{5}{|l|}{ Ethnicity } \\
\hline White & $63.8 \%$ & $83.2 \%$ & $61.1 \%$ & $72.7 \%$ \\
\hline African American & $28.2 \%$ & $11.9 \% \%^{\mathrm{a}}$ & $22.4 \%$ & $13.6 \%$ \\
\hline Asian & $1.2 \%$ & $0.3 \%$ & $2.1 \%$ & $0.0 \%$ \\
\hline Hispanic & $4.0 \%$ & $1.6 \%$ & $0.9 \%$ & $4.5 \%$ \\
\hline Native American & $0.5 \%$ & $0.9 \%$ & $0.2 \%$ & $2.3 \%$ \\
\hline Other & $2.3 \%$ & $2.1 \%$ & $8.3 \%$ & $6.8 \%$ \\
\hline Eligibility via disability & $18.0 \%$ & $40.0 \%{ }^{* * *}$ & $33.0 \%$ & $61.0 \% * * *$ \\
\hline Code for victimization & $1.1 \%$ & $9.1 \% 0^{* * *}$ & $0.1 \%$ & $0 \%$ \\
\hline \multicolumn{5}{|l|}{ Mean days duration of PTSD coding } \\
\hline PTSD only $(n=753)$ & $\mathrm{n} / \mathrm{a}$ & 144 & $\mathrm{n} / \mathrm{a}$ & 104 \\
\hline PTSD + Depression $(n=957)$ & $\mathrm{n} / \mathrm{a}$ & 175 & $\mathrm{n} / \mathrm{a}$ & 324 \\
\hline Complex PTSD $(n=379)^{\mathrm{b}}$ & $\mathrm{n} / \mathrm{a}$ & 285 & $\mathrm{n} / \mathrm{a}$ & 345 \\
\hline \multicolumn{5}{|l|}{ Treatment use } \\
\hline SSRI antidepressant & $18.1 \%$ & $71.5 \% \%^{* * *}$ & $22.6 \%$ & $68.2 \% * * *$ \\
\hline Psychotherapy & $5.0 \%$ & $37.4 \% \%^{* * *}$ & $3.4 \%$ & $20.5 \% \%^{* * *}$ \\
\hline \multicolumn{5}{|l|}{ Comorbidity or associated features } \\
\hline Depression & $9.4 \%$ & $61.7 \% \%^{* * *}$ & $4.8 \%$ & $43.2 \%{ }^{* * *}$ \\
\hline Anxiety & $4.7 \%$ & $30.1 \% 0^{* * *}$ & $2.5 \%$ & $25.0 \% * * *$ \\
\hline Substance abuse & $6.8 \%$ & $23.6 \% \%^{* * *}$ & $1.0 \%$ & $4.5 \%$ \\
\hline Eating disorder & $0.2 \%$ & $2.7 \% 0^{* * *}$ & $0.1 \%$ & $0.0 \%$ \\
\hline Dissociation & $0.3 \%$ & $7.6 \%{ }^{* * *}$ & $0.0 \%$ & $2.3 \%{ }^{* *}$ \\
\hline Somatization & $0.0 \%$ & $0.8 \% \%^{* * *}$ & $0.0 \%$ & $0.0 \%$ \\
\hline Borderline personality disorder & $0.6 \%$ & $13.9 \% * * *$ & $0.2 \%$ & $4.5 \%{ }^{* *}$ \\
\hline \multicolumn{5}{|l|}{ Severe mental illness } \\
\hline Schizophrenia & $3.1 \%$ & $13.4 \% \%^{* * *}$ & $5.1 \%$ & $2.3 \%$ \\
\hline Bipolar disorder & $1.7 \%$ & $16.6 \%^{* * *}$ & $1.8 \%$ & $11.4 \%^{* * *}$ \\
\hline
\end{tabular}

Note. Significance test by Fisher's exact test when cell size is $<5$.

${ }^{a}$ Chi-square test of proportion of African American versus White is significant at $p<.001 .{ }^{b}$ For reproductive age group one-way ANOVA, $F(2,2086)=31.5, p<.001$. Scheffe post hoc test indicates PTSD without depression vs. PTSD with depression difference not significant $(p=.069)$, but contrasts with Complex PTSD are significant at $p<.001$. For postmenopausal group one-way ANOVA, $F(2,41)=3.6$, $p<.05$. Scheffe post hoc test indicates PTSD without depression vs. PTSD with depression contrast is significant at $p=.050$. ${ }^{*} p<.05 .{ }^{* *} p<.01 .{ }^{* * *} p<.001$.

The complex PTSD group's mean duration of PTSD coding was significantly longer by 3 to 4 months $(M=285$ days, $S D=392$ days, respectively; $F(2,2086)=31.5$, $p<.001$, in both comparisons. The rate of suicide or self- inflicted injury coding increased from $0.7 \%$ in the PTSD without depression category to $2.8 \%$ in the PTSD with depression category, and to $12.1 \%$ in the complex PTSD category. 


\section{Disease and Chronic Conditions Among Postmenopausal Women With PTSD}

Only 44 recipients in the postmenopausal-age group had a PTSD diagnosis. The PTSD-diagnosed older women had significantly higher rates of coding than the older comparison group women in the following disease categories after Bonferroni correction for multiple comparisons $(p<$ .003; Pedhazur \& Schmelkin, 1991): digestive (OR=3.7); genitourinary $(\mathrm{OR}=2.6)$; musculoskeletal and connective tissue ( $\mathrm{OR}=5.6$ ); and signs, symptoms, and ill-defined conditions $(\mathrm{OR}=3.4)$. They also had significantly higher rates of chronic pelvic pain $(\mathrm{OR}=6.1)$. (Prevalence rates for all outcomes are available from the corresponding author.) The rest of the results presented pertain to the reproductive-age group.

\section{Disease, Chronic Conditions, and Gynecologic Problems Among Reproductive-Age Women With PTSD}

Among reproductive-aged, PTSD-diagnosed women, coding occurred for every category of disease, all five chronic conditions, and each of the gynecologic health problems at significantly higher rates $(p<.002$ after Bonferroni correction for 23 comparisons). (Prevalence rates are available from the corresponding author.) Bivariate odds ratios in the categories of disease were between 1.3 (blood and blood forming organs) and 4.8 (signs, symptoms, and ill-defined conditions), and they ranged from 2.4 (fibromyalgia) to 4.7 (irritable bowel syndrome) among the chronic conditions in previous research that were associated with a history of sexual abuse or assault. The PTSD-group's mean number of chronic-condition diagnoses was 0.73 (vs. 0.26), $F(1$, $13756)=1050.2, p<.001$. The PTSD group had twice the rate of having one of the chronic-condition diagnoses, 4 times the rate of having two, and 7 and 11 times the rates of having three and four such diagnoses, respectively, a level and distribution of symptomatology which, absent organic findings, could potentially meet diagnostic criteria for somatiform disorders. PTSD-diagnosed women also had increased odds for gynecologic conditions rang- ing from 1.5 for any sexually transmitted infection to 3.4 for dyspareunia, but they had fewer pregnancies $(\mathrm{OR}=$ 0.60). In every health outcome comparison for the categories of diseases and chronic conditions, the prevalence rate was lowest in the reference group (i.e., women with no mental health diagnosis). Within the PTSD-diagnosed group, there also was a consistent dose-response pattern related to PTSD severity.

\section{Multivariate Models of Effects of Insurance Coverage. Victimization, and PTSD Status on Health Outcomes}

We estimated stepwise logistic regression models for all 23 outcomes in the reproductive-age group, estimating first the effect of victimization controlling for exposure to Medicaid coverage. Victimization alone conveyed statistically significantly increased odds for most conditions, after adjusting for exposure to Medicaid coverage (Table 2). When the six-category PTSD variable that distinguished women with depression and complex PTSD was added to these models (using women with no mental health diagnosis as the reference group), it generally weakened the association with victimization and sometimes made the victimization variable lose statistical significance, suggesting that PTSD may sometimes act as a mediator of health outcomes (Baron \& Kenny, 1986). Once PTSD was entered into the model, victimization was not significantly associated with any of the five chronic conditions previously associated with sexual assault or sexual abuse history, except for chronic pelvic pain.

In relation to the six-category case-control variable, the odds ratios for the health outcomes generally followed a dose-response pattern. The PTSD cases without depressive, dissociative, or borderline personality disorder comorbidity ("simple PTSD") are more at risk than the reference category of no mental health diagnosis. Depression without PTSD and simple PTSD vary in terms of which is more strongly associated with the chronic and reproductive conditions. PTSD with depression is usually more strongly associated with ill health than depression alone. The most severe category, complex PTSD, has the strongest 


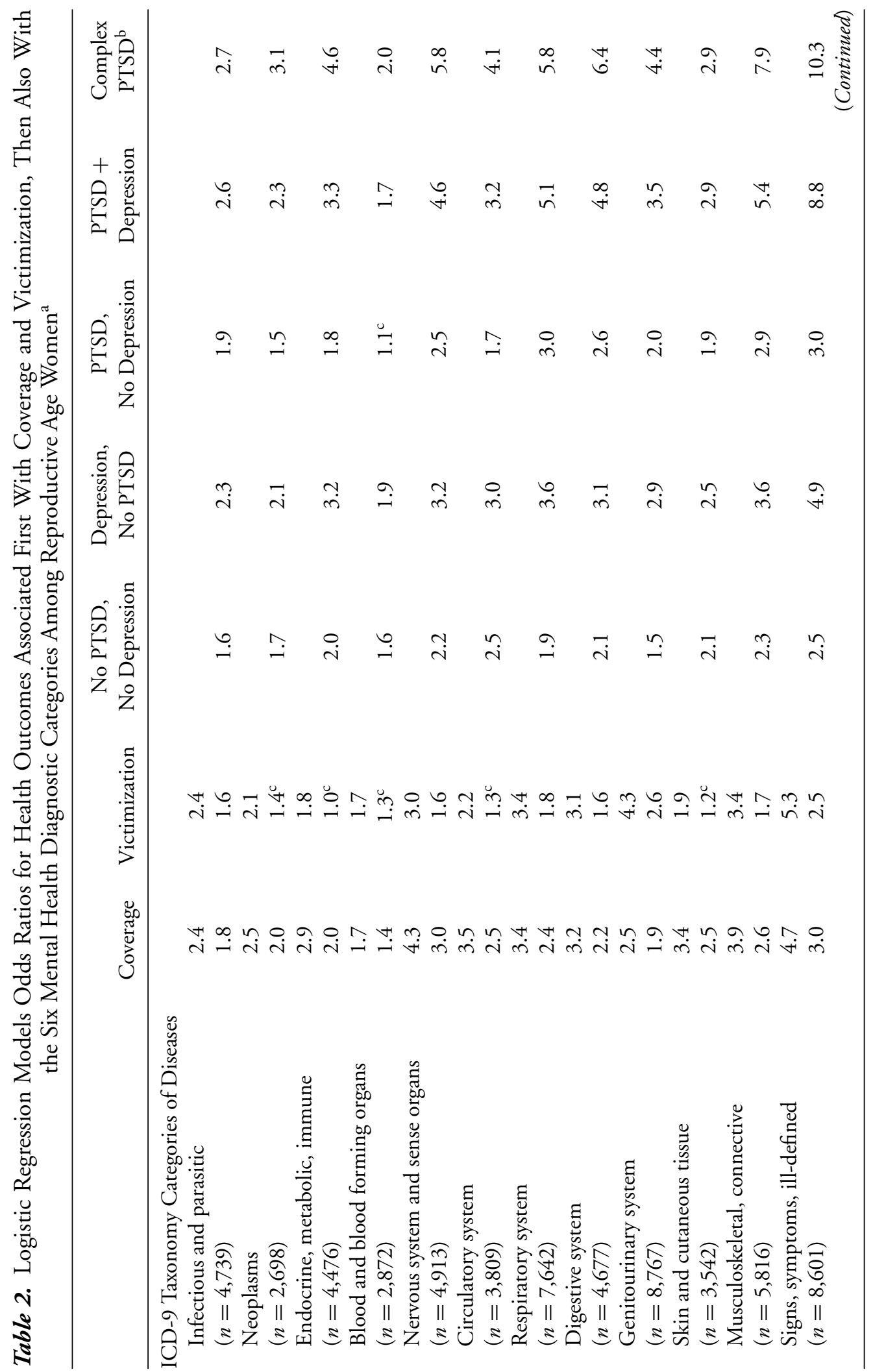




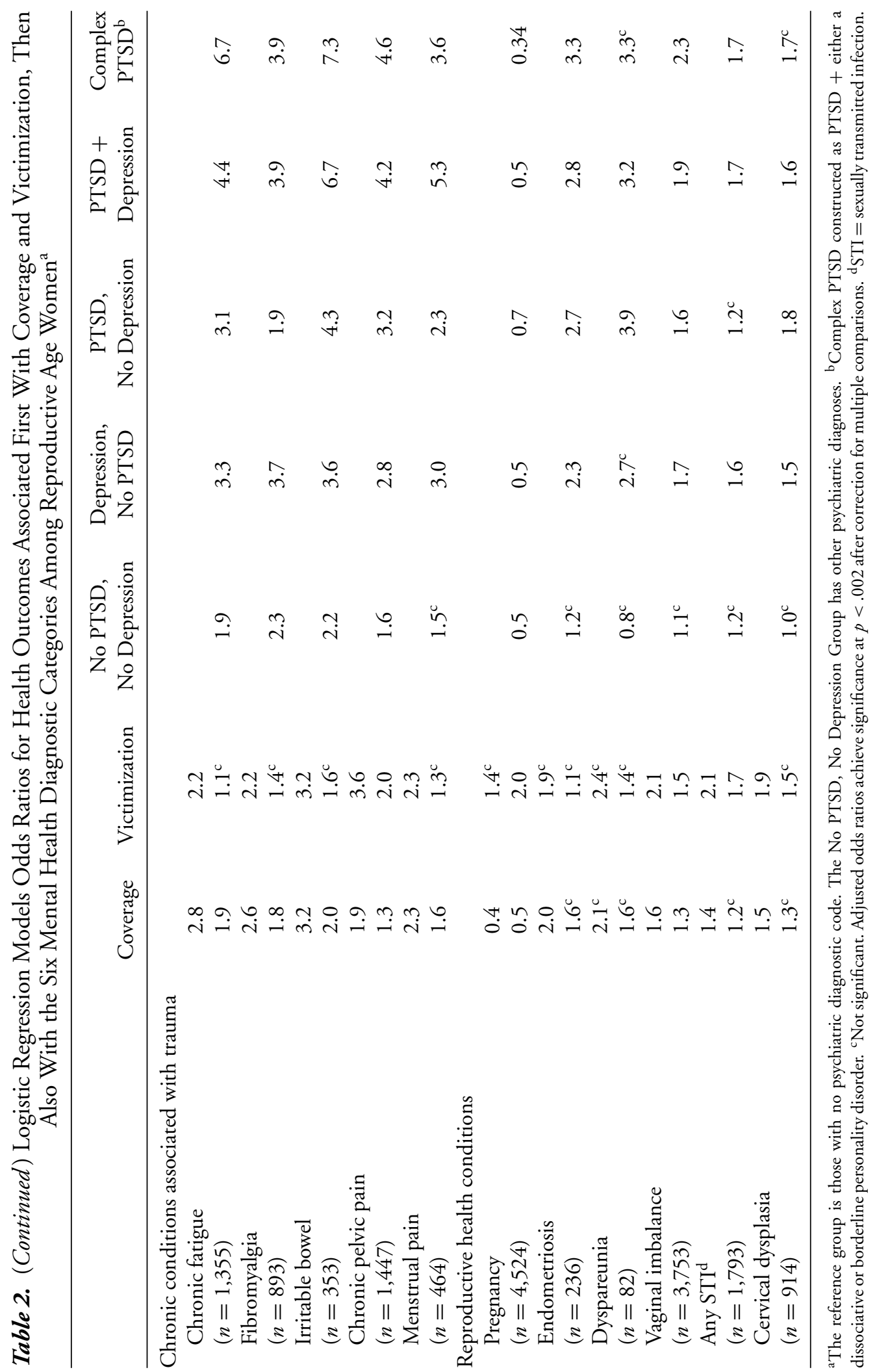


associations with all ICD-9 disease categories except skin disorders. Among the chronic conditions associated with previous sexual assault or sexual abuse, irritable bowel and chronic pelvic pain are associated with PTSD, and chronic fatigue may be associated with PTSD, but fibromyalgia and menstrual pain appear to be more strongly associated with depression. Endometriosis and dyspareunia are associated with PTSD and not victimization, but the relationships with PTSD and the other gynecological conditions are less clear. The pregnancy rate is negatively related to PTSD severity (or to PTSD treatment). Overall, the multivariate models suggest that the extent to which PTSD, depression, and victimization are associated with adverse health outcomes varies, and differing behavioral and biological pathways may be implicated. For the majority of conditions, PTSD in its chronic, psychiatrically comorbid, complex forms increases risk for health problems above and beyond the risk contributed by either victimization or depressiontwo factors already considered to be seriously detrimental to women's health across the life span.

\section{DISCUSSION}

The results of this descriptive epidemiological analysis indicate that PTSD was associated with adverse health outcomes throughout adulthood among female Medicaid recipients, including indication of greater disease morbidity at an earlier age among postmenopausal recipients. This analysis found, similar to many other studies, that victimization increased risk for adverse health outcomes, but it also showed that PTSD appeared to be a stronger predictor than victimization for nearly all of these conditions. Findings from earlier, primarily survey research studies have shown that women with PTSD have more symptoms, worse health perceptions, and more functional disability. This descriptive epidemiological analysis of publicly insured women with a PTSD diagnosis extends this research by showing that (a) this pattern applies to conditions for which women seek care and receive diagnoses in health care settings and that (b) physical comorbidity occurs in a dose-response relationship with PTSD severity and chronicity.

\section{Limitations}

The prevalence of PTSD diagnosis in these data $(0.5 \%)$ is far below the $4.6 \%$ rate of PTSD estimated from an epidemiological study of U.S. women from roughly the same time period (Resnick et al., 1993). Reasons for underdiagnosis cannot be discerned from administrative data. Because of this underdiagnosis, many women with PTSD may be in the comparison group, including the group with depression. Thus, these results may overestimate the effect of depression alone and underestimate the importance of PTSD, and the pattern of health outcomes associated with PTSD could be somewhat different. Because most of the PTSD group was in psychotherapy or using a selective serotonin reuptake inhibitor, these results also may underestimate the effects on health of PTSD that is untreated or the effect of PTSD on medical care seeking among women who do not have a relationship with a mental health provider.

These findings must be considered to be preliminary in nature due to limitations inherent in service-use data and cross-sectional analysis. Rates of hospital coding for victimization are lower than rates of actual occurrence of victimization, and other trauma exposures not considered in this analysis also could be the antecedent for PTSD. PTSD itself was likely underdiagnosed. Thus, the relative magnitude and specific patterns of trauma exposure and PTSD in relation to physical health problems could differ in studies with more comprehensive means of measuring both trauma-exposure history and PTSD. Some cases of PTSD may have been diagnosed only subsequent to experiences of medical traumatic events (e.g., breast cancer diagnosis and/or treatment), introducing a proportion of error in inference about causality that could be reduced in designs where the nature of the antecedent traumatic event can be assessed. Coding errors occur, and their proportion cannot be estimated; however, this error likely would be random. Services not paid for with Medicaid coverage will not be noted in this analysis. Administrative data show actual patterns of utilization without differentiating tentative from confirmed diagnoses. Future studies with physical examination and laboratory data will be needed 
to understand patterns of confirmed diagnoses and their biological mechanisms.

The limitations of using naturalistic data in an exploratory study are balanced by the lack of the sorts of error and bias that can occur when data are collected in the context of a research study. Patterns can be seen across a broad array of conditions, and conclusions are based on actual patient care seeking and clinician practice. Inference from this epidemiological, secondary analysis is strengthened by the number of records included, by the fact that all PTSD cases were used rather than a sample of them, and by comparing PTSD-diagnosed women with all others rather than only with (psychiatrically) healthy controls.

\section{Generalizability}

Overall, according to 2000 Census data (U.S. Census Bureau, n.d., http://factfinder.census.gov), Michigan's sociodemographic profile is similar to the U.S. population with regard to gender, age, educational attainment, and workforce participation. Findings from Michigan Medicaid data can reasonably be generalized to the U.S. population of Medicaid recipients because the state's eligibility, enrollment, and demographic profile in the 1990s was very similar to that of the aggregate U.S. Medicaid program (Health Care Financing Administration, 1996, 1997; Winterbottom, Liska, \& Obermaier, 1995). These findings may not be generalizable to insured women; however, in HMO studies, privately insured traumatic-event survivors also have been found to have higher utilization rates and costs (Walker et al., 2003). In one study that compared medical care seeking by insurance status, privately insured women with a sexual-assault history sought care at slightly higher levels than their Medicaid-insured counterparts (59.4\% privately insured vs. 54.4\% Medicaid-insured 6-month medical service utilization rate; Golding, Stein, Siegel, Burnam, \& Sorenson, 1988). Replication of this analysis with insurance industry data would be useful.

It is more difficult to determine if rates of victimization are representative. With regard to current violent-crime exposures, the Michigan annual rate was 10\% higher than the U.S. rate in 2003 , but both rates equate to roughly
$0.5 \%$ per year (511 vs. 475 cases, respectively, of murder, forcible rape, robbery, or aggravated assault per 100,000 according to FBI statistics [The Henry J. Kaiser Family Foundation, 2003]). The overall rate in these data among the 18- to 54-year-olds for codes for hospital encounters for rape and inflicted injury for the 4-year period was 2.3\%, suggesting underdetection. Low-income women could be expected to live in more high-crime environments, but not all violent crimes result in hospital encounters.

This sample also is distinguished by high rates of disability in the PTSD group. Michigan and U.S. rates of self-reported disability in 2000 Census data (U.S. Census Bureau, n.d.) for all people age 5 years and older are similar (18.7 and $19.3 \%$, respectively), with 5.2 versus $4.8 \%$, respectively, reporting mental disability and 10.7 versus $11.9 \%$, respectively, reporting employment disability. In the 16- to 64-year census age group, disabled people were twice as likely (18.8 vs. $9.6 \%$, respectively, to live below the federal poverty level, making them eligible for Medicaid coverage (Waldrop \& Stern, 2003). In the Michigan Medicaid data used for this analysis, $21.6 \%$ of the women overall were eligible for Medicaid due to disability, with $18 \%$ in the comparison group and $40 \%$ in the PTSD group. From these data, it is not possible to know the extent to which the PTSD group's higher rate reflects greater mental health disability, greater physical disability from traumatic events that also increase risk for PTSD (e.g., motor vehicle accidents, fires, life-threatening illness, and crime), or both.

Finally, clinician diagnosis of PTSD in these data would appear to underdiagnose PTSD to an extent that it could plausibly be only the most severe cases that are identified. If this were the case, this source of error could result in Type I error (i.e., concluding that PTSD is associated with adverse health outcomes when the relationship really only occurs with severe PTSD). Use of the six-category mental health variable provides information to show that a range of severity of PTSD cases did appear to be identified. This increases support for the general conclusion that PTSD is associated with these health conditions because a dose-response pattern is visible, with even "simple PTSD" associated with adverse outcomes. Nevertheless, these conclusions 
likely should be considered to apply only to treatmentseeking women with PTSD. Further research with systematic diagnostic procedures and community samples is needed.

\section{Implications}

Clinical research can address the limitations of this descriptive epidemiological analysis by systematically determining traumatic-event exposure, PTSD diagnosis, and psychiatric comorbidity, by including biological indicators of multiple stress systems' functioning, by differentiating tentative from confirmed diagnoses for physical conditions, and by including both clinical and community samples. Future designs also should attempt to capture instances when underutilization from PTSD-related avoidance of appropriate medical care (e.g., pap tests, mammograms, and blood tests) resulted in adverse outcomes (Schnurr \& Green, 2004). Observational studies that measure and model the impacts of psychotherapy, medication, and instances of integrated mental health and medical treatment would provide effect size data for future clinical trials of PTSD-related interventions to improve physical health conditions. Future designs ideally also would consider whether traumatic events and PTSD are causally related to these physical conditions or whether the stress-regulation systems' alterations seen across these conditions are, instead, a shared vulnerability for both PTSD and ill health (Heim, Ehlert, \& Hellhammer, 2000). The relative roles of depression and PTSD in relation to onset of ill health are important to discern in future studies.

Anxiety disorders, including PTSD, have been associated with high levels of medical utilization, cost, disability, and suicide mortality regardless of whether mental health treatment occurred (Greenberg et al., 1999). Findings from this study confirm that primary prevention of trauma exposures and secondary prevention of PTSD are critical priorities to meet both public health and medical cost-containment goals. Focus on the health effects of PTSD may be especially beneficial in populations where trauma exposure and mental health sequelae are most severe, and where case-finding efforts should be routine, including severely mentally ill and substance-dependent women, homeless women, and those connecting with rape crisis and domestic violence programs. It also is evident from this analysis, where PTSD cases were identified most often in mental health settings, that mental health professionals may be able to play an appropriate role in their patients' physical health and medical care. More models for collaborative treatment are needed, as are studies of physical health outcomes of psychological and pharmacological treatments for PTSD. If PTSD were more often recognized as a factor in women's healthcare encounters and treated as early in the life span as possible, then the ill effects on health that were associated in this analysis in a dose-response pattern might be decreased.

\section{REFERENCES}

Agresti, A. (1990). Categorical data analysis. New York: Wiley.

Andreski, P., Chilcoat, H. D., \& Breslau, N. (1998). Posttraumatic stress disorder and somatization symptoms: A prospective study. Psychiatry Research, 79, 131-138.

Baron, R. M., \& Kenny, D. A. (1986). The moderator-mediator variable distinction in social psychological research: Conceptual, strategic, and statistical considerations. Journal of Personality \& Social Psychology, 51, 173-182.

Breslau, N., Davis, G. C., Peterson, E. L., \& Schultz, L. R. (1997). Psychiatric sequelae of posttraumatic stress disorder in women. Archives of General Psychiatry, 54, 81-87.

Breslow, N. E., \& Day, N. E. (1993). Statistical methods in cancer research, Vol. 1: The analysis of case-control studies. Oxford, England: Oxford University Press.

Cohen, J. (1988). Statistical power analysis for the behavioral sciences (2nd ed.). Hillsdale, NJ: Erlbaum.

Golding, J. M., Stein, J. A., Siegel, J. M., Burnam, M. A., \& Sorenson, S. B. (1988). Sexual assault history and use of health and mental health services. American Journal of Community Psychology, 16, 625-644.

Greenberg, P. E., Sisitsky, T., Kessler, R. C., Finkelstein, S. N., Berndt, E. R., Davidson, J. R. T., et al. (1999). The economic burden of anxiety disorders in the 1900's. Journal of Clinical Psychiatry, 60, 427-435.

Greenland, S. (1989). Modeling and variable selection in epidemiologic analysis. American Journal of Public Health, 79, 340-349. 
Health Care Financing Administration. (1996). Medicaid statistics, program, and financial statistics, fiscal year 1994. Baltimore: U.S. Department of Health and Human Services.

Health Care Financing Administration. (1997). Medicare and Medicaid statistical supplement. Baltimore: U.S. Department. of Health and Human Services.

Health Insurance Portability and Accountability Act of 1996, Pub.L.No. 104-191, $\S 1173$ (1996). Retrieved January 24, 2006, from http://www.hhs.gov/ocr/hipaa

Heim, C., Ehlert, U., \& Hellhammer, D. H. (2000). The potential role of hypocortisolism in the pathophysiology of stress-related bodily disorders. Psychoneuroendocrinology, 25, 1-35.

The Henry J. Kaiser Family Foundation. (2003). Violent crime offenses rate per 100,000 population, 2003. Retrieved January 24, 2006, from http://statehealthfacts.org/cgi-bin/healthfacts. cgi?action $=$ compare\&category $=$ Health + Status\&subcategory $=$ Violent + Crime\&topic $=$ Violent + Crime + Offenses\&gsaview $=1$

Hintze, J. L. (2000). PASS 2000 user's guide. Kaysville, UT: NCSS.

Hulley, S. B., Cummings, S. R., Browner, W. S., Grady, D., Hearst, N., \& Newman, T. B. (Eds.). (2001). Designing clinical research (2nd ed., pp. 95-105). Philadelphia: Lippincott Williams \& Wilkins.

Irwin, C., Falsetti, S. A., Lydiard, R. B., Ballenger, J. C., Brock, C. D., \& Brener, W. (1996). Comorbidity of posttraumatic stress disorder and irritable bowel syndrome. Journal of Clinical Psychiatry, 57, 576-578.

Kimerling, R., Clum, F. A., \& Wolfe, J. (2000). Relationships among trauma exposure, chronic posttraumatic stress disorder symptoms, and self-reported health in women: Replication and extension. Journal of Traumatic Stress, 13, 115-128.

Ouimette, P., Cronkite, R., Henson, B. R., Prins, A., Gima, K., \& Moos, R. H. (2004). Posttraumatic stress disorder and health status among female and male medical patients. Journal of Traumatic Stress, 17, 1-9.

Pedhazur, E. J., \& Schmelkin L. P. (1991). Measurement, design, \& analysis: An integrated approach (pp. 485-486). Hillsdale, NJ: Erlbaum.

Practice Management Information Corporation. (1998). International classification of diseases (9th rev.), Clinical modification (5th ed.). Los Angeles: Author.
Rasmusson, A. M., \& Friedman, M. J. (2002). Gender issues in the neurobiology of PTSD. In R. Kimerling, P. Ouimette, \& J. Wolfe (Eds.), Gender \& PTSD (pp. 43-75). New York: Guilford Press.

Rasmusson, A. M., Lipshitz, D. S., Wang, S., Hu, S., Vojvoda, D., Bremner, J. D., et al. (2001). Increased pituitary and adrenal reactivity in postmenopausal women with posttraumatic stress disorder. Biological Psychiatry, 50, 965-977.

Resnick, H. S., Kilpatrick, D. G., Dansky, B. S., Saunders, B. E., \& Best, C. L. (1993). Prevalence of civilian trauma and posttraumatic stress disorder in a representative national sample of women. Journal of Consulting Clinical Psychology, 61, 984-991.

Schnurr, P. P., \& Green, B. L. (2004). Understanding the relationships among trauma, posttraumatic stress disorder, and health outcomes In P. P. Schnurr \& B. L. Green (Eds.), Trauma and health: Physical health consequences of exposure to extreme stress (pp. 247-276). Washington, DC: American Psychological Association.

Seng, J. S., Graham-Bermann, S. A., Clark, M. K., McCarthy, A. M., \& Ronis, D. L. (2005). Posttraumatic stress disorder and physical comorbidity among female children and adolescents: Results from service-use data. Pediatrics, 116(6), e767776.

Seng, J. S., Kohn-Wood, L. P., \& Odera, L. (2005). Exploring racial disparity in posttraumatic stress disorder diagnosis: Implications for care of African American women. Journal of Obstetric, Gynecologic, and Neonatal Nursing, 34, 521-530.

U.S. Census Bureau. (n.d.). 2000 Census Factfinder data for the State of Michigan. Retrieved December 28, 2004, from http://www.factfinder.census.gov

Waldrop, J., \& Stern, S. M. (2003). Disability status: 2000. U.S Census brief C2KBR-17. Retrieved December 28, 2004, from http://www.census.gov/prod/2003pubs/c2kbr-17.pdf

Walker, E. A., Katon, W., Russo, J., Ciechanowski, P., Newman, E., \& Wagner, A. W. (2003). Health care costs associated with posttraumatic stress disorder symptoms in women. Archives of General Psychiatry, 60, 369-374.

Winterbottom, C., Liska, D. W., \& Obermaier, K. M. (1995). Statelevel databook on health care access and financing (2nd ed. \& Suppl.). Washington, DC: The Urban Institute. 